\title{
Taxas de mortalidade por câncer corrigidas para os idosos dos estados do Nordeste brasileiro
}

\author{
Corrected cancer mortality rates for the elderly in the states of the \\ Brazilian northeast
}

João Batista Carvalho (https://orcid.org/0000-0001-7385-8348) ${ }^{1}$

Neir Antunes Paes (https://orcid.org/0000-0002-0927-9103) ${ }^{1}$

${ }^{1}$ Unidade Acadêmica de Estatística, Centro de Ciências e Tecnologia, Universidade Federal de Campina Grande. R. Aprígio Veloso 882, Bela Vista. 58429-900. Campina Grande PB Brasil.

joaouaest@gmail.com

\begin{abstract}
Estimates of the true magnitude of mortality in the Brazilian northeast has been fraught with major obstacles due to underreporting and problems with the quality of information on the basic causes of death. The scope of this study was to evaluate the impact of the redistribution of deaths corrected by Active Search Research and Garbage codes on the mortality rates of the major types of cancer among the elderly in the northeastern states. The Ledermann method was used to reallocate the deaths. The number of deaths before and after the correction revealed a significant variation, especially the figures for breast cancer where the variation was $53.5 \%$. With corrected data, the elderly aged 80 years or older had a prostate cancer death rate which was 18 times higher than those aged 60 to 64 years. Higher levels of prostate cancer deaths among men, and breast cancer among women, were observed in all of the northeastern states. Tracheal, bronchial, lung and stomach cancer resulted in different levels of distribution according to gender, with higher rates among men than among women. Correction of death data resulted in significant increases in the number of recorded deaths. The methodology adopted in this work was the simple application of feasible factual data by the information system managers.

Key words Mortality, Cancer, Elderly, Brazilian northeast, Quality of the data
\end{abstract}

Resumo As estimativas das reais magnitudes da mortalidade no Nordeste têm enfrentado importantes entraves devido ao subregistro e aos problemas na qualidade das informações sobre as causas básicas de óbito. Teve-se como objetivo avaliar o impacto da redistribuição dos óbitos corrigidos pela Pesquisa de Busca Ativa e de códigos garbage nas taxas de mortalidade dos principais cânceres de idosos dos estados do Nordeste. Utilizou-se o método de Ledermann para redistribuir os óbitos. O número de óbitos antes e após a correção apresentou uma variação expressiva, com destaque para o câncer de mama cuja variação foi de 53,5\%. Com os dados corrigidos, idosos com 80 anos ou mais apresentaram uma taxa de mortalidade por câncer de próstata 18 vezes maior que os de 60 a 64 anos. Foram observados niveis mais elevados de mortes por câncer de próstata para homens e câncer de mama para as mulheres em todos os estados do Nordeste. O câncer da traqueia, brônquios e pulmões e o câncer de estômago resultaram em níveis de distribuição diferentes segundo sexo, com taxas mais elevadas nos homens que nas mulheres. A correção dos dados de óbitos significou importantes acréscimos na quantidade de óbitos registrados. A metodologia adotada neste trabalho é de simples aplicação, factivel de uso pelos gestores dos sistemas de informação.

Palavras-chave Mortalidade, Câncer, Idosos, Nordeste, Qualidade dos dados 


\section{Introdução}

Os crescentes níveis da mortalidade por câncer o posicionam como a segunda principal causa de morte no mundo e, em muitos países pobres e em desenvolvimento, as projeções sinalizam que ocupará o primeiro lugar nas próximas décadas ${ }^{1}$. De acordo com dados do projeto Global Cancer (GLOBOCAN 2012), realizado pela Organização Mundial da Saúde, o câncer foi responsável por 8,2 milhões de mortes (22\% de todas as mortes por doenças não transmissíveis) em 2012. Dessas, cerca de 4,7 milhões (57,3\%) e 3,5 milhões $(42,7 \%)$ foram de homens e mulheres, respectivamente ${ }^{2,3}$.

Ocupando o segundo lugar no Brasil, as neoplasias foram responsáveis por 209.780 (16,6\%) óbitos em 2015. Desses óbitos, 68,4\% ocorreram em pessoas de 60 anos ou mais, que constitui o grupo que recebe a maior carga de mortalidade por neoplasias. Essa segunda posição foi atingida a partir do ano 2003, cujo número de óbitos foi de 134.691 com os idosos respondendo por $63,7 \%$ do total ${ }^{4}$.

A participação dos idosos na mortalidade geral é cada vez maior no Brasil devido ao envelhecimento populacional, desencadeado a princípio nos anos 60, quando os primeiros sinais da redução da fecundidade foram dados e com uma tendência irreversível desde então. O envelhecimento da população é um fenômeno inédito na história do País, que vem trazendo modificações importantes na estrutura etária com repercussões em vários aspectos da vida ${ }^{5}$.

De acordo com estimativas reportadas no Relatório Mundial sobre Envelhecimento e Saúde, havia 841 milhões de idosos com 60 anos ou mais no mundo e 24,4 milhões no Brasil em 2015. Nesse ano, a porcentagem de idosos era de $12,5 \%$ no País e, segundo projeções, deve alcançar 30\% na metade deste século. O fenômeno do envelhecimento populacional se alastrou em todo o País, com reflexo importante na Região Nordeste ${ }^{6}$.

Estudo desenvolvido por Barbosa et al. ${ }^{7}$ apontou tendências de aumento nas taxas de mortalidade por câncer em ambos os sexos até o ano de 2030, para as regiões Norte e Nordeste, enquanto que para as demais regiões brasileiras tendências decrescentes nessas taxas foram previstas. $\mathrm{O}$ estudo também concluiu que as acentuadas diferenças regionais e por sexo existentes na mortalidade por câncer no Brasil continuarão a aumentar até o ano de 2030. Neste ano, as projeções aponta- ram que a Região Nordeste apresentará a mais alta mortalidade por câncer do Brasil.

Entretanto, uma questão que antecede o cálculo da magnitude das taxas de mortalidade nas regiões menos desenvolvidas do País é a crítica sobre a qualidade de suas estatísticas vitais. Baseado nas regras da Classificação Internacional de Doenças, a morte do indivíduo deve ser atribuída a uma única causa, e ela deve ser a causa básica que desencadeou o óbito. Embora com magnitude variável, a não identificação da causa básica do óbito ainda ocorre regularmente nos sistemas de registros civis dos estados brasileiros ${ }^{8-10}$. Assim, uma proporção considerável de mortes é atribuída aos chamados códigos garbage (CG), que incluem as causas inespecíficas ("mal definidas") dentro de Capítulos da CID-10 ${ }^{11,12}$.

Adicionalmente, o subregistro de óbitos é outro fator que compromete diretamente a qualidade dos indicadores de mortalidade como proporções de óbitos e taxas de mortalidade e, consequentemente, a tomada de decisão por parte dos gestores de políticas de saúde pública. Assim, quando as estatísticas vitais são corrigidas para prováveis erros de codificação, quadros substancialmente diferentes podem surgir ${ }^{10,13,14}$.

Uma das iniciativas do Ministério da Saúde com vistas à melhoria na qualidade das estatísticas vitais foi a realização da Pesquisa de Busca Ativa de Óbitos e Nascimentos a partir do ano 2010, através da qual foram recuperados óbitos que não haviam sido registrados no Sistema de Informações sobre Mortalidade (SIM) e identificadas a verdadeira causa básica para um número expressivo de óbitos que haviam sido classificados na categoria de mal definidos. Pela primeira vez essa pesquisa foi realizada em larga escala no Brasil, abrangendo a Região Nordeste e a Amazônia Legal ${ }^{15}$.

Embora existam estudos que abordem a questão da correção do subregistro e a redistribuição de óbitos por causas mal definidas no Brasil ${ }^{8,16,17}$, poucos são aqueles em que as mortes designadas aos códigos garbage são levadas em conta na correção de erros de codificação. Assim, pouco se sabe sobre os efeitos que as correções acarretariam nas magnitudes e tendências da mortalidade. Neste contexto, teve-se como objetivo avaliar o impacto da redistribuição dos óbitos corrigidos pela Pesquisa de Busca Ativa e de códigos garbage nas taxas de mortalidade dos principais cânceres de idosos dos estados do Nordeste. 


\section{Métodos}

Trata-se de um estudo ecológico com corte transversal no ano 2010 relacionado à região Nordeste, tendo como unidades de análise observacional seus nove estados. Com uma abordagem quantitativa, fez-se uso de informações de óbitos registrados no SIM e dos dados sobre população do Censo Demográfico do IBGE, desagregados por sexo e faixa etária de idosos a partir dos 60 anos.

Para reduzir o efeito das flutuações aleatórias nos dados de óbitos, utilizou-se a média dos óbitos computados pela Pesquisa de Busca Ativa para o triênio 2009, 2010 e 2011. Os dados mais recentes dessa pesquisa se referem a 2013. No entanto, o cálculo das taxas de mortalidade para esse ano exigiria projeções da população, incorporando, assim, mais erros nas estimativas. Desse modo, devido à proximidade desse ano com o triênio citado e a disponibilidade da população censitária de 2010, optou-se em utilizar 2010, em vez 2013, como ano de referência.

Neste estudo, foram selecionados os três principais tipos de câncer responsáveis pelo maior número de mortes de idosos do Nordeste em $2010^{4}$. Assim, para os homens, foram selecionados o câncer de próstata (código C61), da traqueia, brônquios e pulmões (C33-C34) e de estômago (C16). Estes dois últimos junto com o câncer de mama (C50) compõem os selecionados para as mulheres. Esses tipos de câncer foram responsáveis por $46 \%$ e $25 \%$ das mortes por câncer de homens e mulheres da população de 60 anos ou mais em 2010, respectivamente. As etapas traçadas para o desenvolvimento deste trabalho são descritas a seguir.

$1^{\text {a }}$ Etapa: para reduzir o impacto de déficit de cobertura e classificação de óbitos no grupo de causas mal definidas da CID-10 foram utilizados os dados de óbitos por câncer corrigidos segundo a Pesquisa de Busca Ativa para cada estado do Nordeste ${ }^{15}$.

2a Etapa: aos óbitos atribuídos ao câncer pela Pesquisa de Busca Ativa foram adicionados os óbitos referentes aos códigos garbage do Tipo 4 específicos do câncer, de acordo com a tipologia criada por Naghavi et al. ${ }^{12}$. Os códigos garbage selecionados para análise foram: neoplasias malignas de outras localizações e mal definidas (C76) e neoplasias malignas sem especificação de localização (C80).

$3^{\text {a }}$ Etapa: os óbitos resultantes da segunda etapa foram redistribuídos para os tipos de câncer utilizando o método proposto por Ledermann ${ }^{18}$.
Uma vantagem do método desenvolvido por Ledermann em relação ao tradicional método de redistribuição proporcional é que a variação dos óbitos antes e após a redistribuição não é constante para os tipos de câncer. Este método faz uso da regressão linear simples. Em sua aplicação foi considerada como variável resposta a proporção de óbitos dos principais tipos de câncer e como covariável a proporção de óbitos por causas mal definidas de cada estado da região Nordeste, desagregada por sexo.

Em formalidade matemática, seja $Y_{i j}$ a variável que representa a proporção de óbitos do $i$-ésimo estado do Nordeste atribuído ao $j$-ésimo tipo de câncer e seja $\mathrm{X}_{\mathrm{i}}$ a proporção de óbitos por causas mal definidas atribuída ao câncer, referente ao $i$-ésimo estado. A $j$-ésima equação de regressão linear de $\mathrm{X}_{\mathrm{i}}$ sobre $\mathrm{Y}_{\mathrm{ij}}$ estimada é dada por:

$$
\hat{\mathrm{Y}}_{\mathrm{ij}}=a_{j}+b_{j} \mathrm{X}_{\mathrm{i}}
$$

Em que $a_{j}$ e $b_{j}$ são os coeficientes linear e angular, respectivamente, obtidos pelo método de mínimos quadrados ordinários. Pode ser demonstrado que $\sum_{j=1}^{\mathrm{r}} \mathrm{a}_{\mathrm{j}}=0$ e $\sum_{\mathrm{j}=1}^{\mathrm{r}} \mathrm{b}_{\mathrm{j}}=-1$, em que $r$ é o número de estados. Em geral, espera-se uma relação linear inversa entre a proporção de óbitos pelas causas mal definidas e os grupos de causas bem definidos. Assim, é razoável que $-1<b_{j}<0$.

A equação de redistribuição dos óbitos mal definidos tem a seguinte expressão:

$$
O_{i j}=-\mathrm{b}_{\mathrm{j}} \mathrm{W}_{\mathrm{i}}+\mathrm{Z}_{\mathrm{ij}}
$$

Em que,

$O_{i j}$ : óbitos atribuídos ao $j$-ésimo tipo de câncer para a $i$-ésimo estado após a redistribuição das causas mal definidas;

$\mathrm{b}_{j}$ : coeficiente angular da reta de regressão de Ledermann, expresso em proporção;

$\mathrm{W}_{\mathrm{i}}$ : óbitos por causas mal definidas referente ao $i$-ésimo estado;

$Z_{\mathrm{ij}}$ : óbitos observados do $i$-ésimo estado atribuído ao j-ésimo tipo de câncer.

4a Etapa: com os dados registrados e os corrigidos, calculou-se a variação para os principais tipos de câncer em cada estado do Nordeste. Fez-se a redistribuição dos óbitos corrigidos totais para cada faixa etária quinquenal de idosos através do critério usual da proporcionalidade.

5a Etapa: foram obtidas as taxas de mortalidade específicas e, a partir destas, as taxas de mortalidade geral para os tipos de câncer foram padronizadas para cada estado utilizando como 
população padrão a população idosa do Nordeste, referente a ambos os sexos e retroprojetada para o meio do ano de 2010. As taxas foram expressas por 100.000 habitantes. Considerou-se também o cálculo da amplitude total das taxas entre os estados.

Por se tratar de dados secundários provenientes de bancos de dados de domínio público, disponibilizados eletronicamente, justifica-se o não encaminhamento deste estudo para aprovação por comitê de ética em pesquisa.

\section{Resultados}

De acordo com o método de Ledermann, do total de 3.403 óbitos mal definidos referentes aos homens idosos em 2010 a ser redistribuído entre os tipos de câncer, $1.312(38,6 \%)$ corresponderam aos três principais, sendo que $652(19,2 \%)$ foram atribuídos ao câncer de próstata, $531(15,6 \%)$ ao câncer da traqueia, brônquios e pulmões e 129 $(3,8 \%)$ ao câncer de estômago. Em relação às mulheres, de um total de 2.826 óbitos mal definidos, $1.123(39,7 \%)$ foram designados aos cânceres de mama, da traqueia, brônquios e pulmões e de estômago, que corresponderam a 22,6\%, 12,7\% e $4,4 \%$ dos óbitos mal definidos redistribuídos entre os cânceres femininos, respectivamente.

Concluídas as etapas da redistribuição, o número de óbitos antes e após a correção apresentou variação expressiva em todos os casos (Tabela 1). O número de óbitos de câncer de próstata aumentou $19,5 \%$. O câncer de mama apresentou a maior variação $(53,5 \%)$. O aumento no número de óbitos por câncer de traqueia, brônquios e pulmões ficou em torno de 33\% em ambos os sexos. Já para o câncer de estômago, a variação foi menor, 10,2\% para os homens e 17,3\% para as mulheres.

Verificou-se uma maior contribuição da Pesquisa de Busca Ativa na variação total dos óbitos corrigidos em ambos os sexos, comparado com a contribuição dos CG. No conjunto dos principais tipos de câncer estudados para os homens, a contribuição da Pesquisa de Busca Ativa foi de $17,3 \%$ e dos CG de 3,9\%, enquanto que para as mulheres os percentuais de ganho foram, respectivamente, $28,2 \%$ e $9,4 \%$.

Observa-se que as taxas padronizadas de mortalidade aumentaram com a idade para o Nordeste, cujo padrão foi verificado em todos os tipos de câncer analisados (Tabela 2). Ressalta-se o impacto da idade nas taxas de câncer de próstata cujo valor foi 17,9 vezes maior, saltando de 31,1 na faixa etária de 60 a 64 anos para 557,4 na faixa etária de 80 e mais após a correção. Para os demais tipos de câncer, este aumento não ultrapassou 3,5 vezes, que corresponde ao câncer de estômago feminino. Nota-se também que, embora diferissem entre os tipos de câncer, as variações das taxas segundo faixa etária foram muito próximas dentro de cada tipo de câncer.

Entre as mulheres idosas, o câncer de mama apresentou as maiores taxas de mortalidade por idade. Para os homens a partir dos 70 anos, foram observados níveis mais elevados de morte por câncer de próstata. Abaixo dessa idade, os níveis de mortalidade por câncer de traqueia, brônquios e pulmões ainda foram os maiores na população idosa masculina.

Na Tabela 3, verifica-se que o câncer de próstata foi o responsável pela maior parcela das mortes dos idosos, apresentando as maiores taxas de mortalidade entre os tipos de câncer em todos os estados do Nordeste. Dentre estes, Sergipe apresentou a maior taxa e Maranhão, a menor. A amplitude total (AT) dessas taxas, após a correção, foi de 90,6 óbitos por 100.000 habitantes.

O câncer da traqueia, brônquios e pulmão e o câncer de estômago resultaram em níveis de distribuição diferentes segundo sexo, com taxas mais elevadas nos homens idosos que nas mulheres idosas. Em nível regional, as taxas por câncer da traqueia, brônquios e pulmão foram maiores nos estados do Ceará, Pernambuco e Rio Grande do Norte. Estes estados e o estado da Paraíba apresentaram as maiores taxas de mortalidade por câncer de estômago. Maranhão, Piauí e Alagoas estiveram entre os de menores taxas para os tipos de câncer considerados.

Com a correção e redistribuição dos óbitos, em ambos os sexos, os estados do Maranhão e Bahia apresentaram as mais elevadas variações nas taxas de mortalidade por câncer de próstata, mama e traqueia, brônquios e pulmões. Por outro lado, para estes mesmos tipos de câncer e em ambos os sexos, os estados de Sergipe e Pernambuco estiveram entre os estados com as menores variações. A variação das taxas de mortalidade por câncer de estômago foi mais expressiva nos estados da Bahia e Sergipe, em ambos os sexos.

\section{Discussão}

Embora a discussão sobre os níveis de cobertura dos óbitos no Nordeste possa levar à controvérsias, pelo menos dois consensos podem ser destacados: a melhoria na cobertura ao longo dos anos e que o subregistro ainda é preocupante $e^{8,9,17,19}$. 
Tabela 1. Contribuição da Busca Ativa (BA) e códigos garbage (CG) na correção dos óbitos por tipo de câncer, segundo sexo. Nordeste, 2010.

\begin{tabular}{|c|c|c|c|c|c|c|c|c|c|}
\hline \multicolumn{5}{|c|}{ Homens } & \multicolumn{5}{|c|}{ Mulheres } \\
\hline \multirow{2}{*}{ Antes } & \multirow{2}{*}{ Após } & \multicolumn{3}{|c|}{ Variação (\%) } & \multirow{2}{*}{ Antes } & \multirow{2}{*}{ Após } & \multicolumn{3}{|c|}{ Variação (\%) } \\
\hline & & BA & CG & Total & & & BA & CG & Total \\
\hline \multicolumn{5}{|c|}{ Próstata } & \multicolumn{5}{|c|}{ Mama } \\
\hline 3.337 & 3.989 & 16,0 & 3,5 & 19,5 & 1.193 & 1.831 & 40,1 & 13,3 & 53,5 \\
\hline \multicolumn{10}{|c|}{ Traqueia, brônquios e pulmões } \\
\hline 1.590 & 2.121 & 27,3 & 6,1 & 33,4 & 1.070 & 1.430 & 25,2 & 8,4 & 33,6 \\
\hline \multicolumn{10}{|c|}{ Estômago } \\
\hline 1.266 & 1.395 & 8,3 & 1,9 & 10,2 & 722 & 847 & 13,0 & 4,3 & 17,3 \\
\hline \multicolumn{10}{|c|}{ Total } \\
\hline 6.193 & 7.505 & 17,3 & 3,9 & 21,2 & 2.985 & 4.108 & 28,2 & 9,4 & 37,6 \\
\hline
\end{tabular}

Tabela 2. Número de óbitos e taxas específicas de mortalidade por tipo de câncer antes e após a correção, segundo sexo e faixa de idade. Nordeste, 2010.

\begin{tabular}{|c|c|c|c|c|c|c|c|c|c|c|}
\hline \multirow{4}{*}{ Idade } & \multicolumn{5}{|c|}{ Homens } & \multicolumn{5}{|c|}{ Mulheres } \\
\hline & \multicolumn{2}{|c|}{ Antes } & \multicolumn{2}{|c|}{ Após } & \multirow{2}{*}{$\frac{\text { Variação }}{\text { da taxa }(\%)}$} & \multicolumn{2}{|c|}{ Antes } & \multicolumn{2}{|c|}{ Após } & \multirow{2}{*}{$\frac{\text { Variação }}{\text { da taxa }(\%)}$} \\
\hline & $\mathbf{n}$ & taxa & $\mathbf{n}$ & $\operatorname{taxa}$ & & $\mathbf{n}$ & taxa & $\mathbf{n}$ & taxa & \\
\hline & \multicolumn{5}{|c|}{ Próstata } & \multicolumn{5}{|c|}{ Mama } \\
\hline $60-64$ & 197 & 25,9 & 236 & 31,1 & 20,1 & 258 & 29,4 & 396 & 45,1 & 53,4 \\
\hline $65-69$ & 335 & 58,0 & 402 & 69,5 & 19,8 & 231 & 33,7 & 354 & 51,8 & 53,7 \\
\hline $70-74$ & 522 & 117,0 & 626 & 140,4 & 20,0 & 226 & 40,7 & 346 & 62,3 & 53,1 \\
\hline $75-79$ & 611 & 212,6 & 730 & 253,9 & 19,4 & 160 & 42,7 & 248 & 66,1 & 54,8 \\
\hline $80 \mathrm{e}+$ & 1672 & 466,8 & 1996 & 557,4 & 19,4 & 317 & 62,7 & 486 & 96,0 & 53,1 \\
\hline \multirow[t]{2}{*}{ Total } & 3337 & 137,4 & 3989 & 164,2 & 19,5 & 1192 & 39,8 & 1830 & 61,0 & 53,3 \\
\hline & \multicolumn{10}{|c|}{ Traqueia, brônquios e pulmões } \\
\hline $60-64$ & 290 & 38,1 & 387 & 50,9 & 33,6 & 202 & 23,1 & 270 & 30,8 & 33,3 \\
\hline $65-69$ & 341 & 59,1 & 456 & 79,0 & 33,7 & 220 & 32,2 & 293 & 42,9 & 33,2 \\
\hline $70-74$ & 344 & 77,2 & 459 & 102,9 & 33,3 & 226 & 40,7 & 303 & 54,5 & 33,9 \\
\hline $75-79$ & 257 & 89,5 & 344 & 119,8 & 33,9 & 170 & 45,2 & 227 & 60,4 & 33,6 \\
\hline $80 \mathrm{e}+$ & 358 & 99,9 & 476 & 132,8 & 32,9 & 252 & 49,7 & 336 & 66,5 & 33,8 \\
\hline \multirow[t]{2}{*}{ Total } & 1590 & 65,5 & 2122 & 87,3 & 33,3 & 1070 & 35,7 & 1429 & 47,7 & 33,6 \\
\hline & \multicolumn{10}{|c|}{ Estômago } \\
\hline $60-64$ & 232 & 30,6 & 256 & 33,7 & 10,1 & 109 & 12,4 & 128 & 14,6 & 17,7 \\
\hline $65-69$ & 247 & 42,8 & 273 & 47,2 & 10,3 & 127 & 18,6 & 150 & 21,9 & 17,7 \\
\hline $70-74$ & 252 & 56,4 & 277 & 62,2 & 10,3 & 139 & 25,0 & 162 & 29,2 & 16,8 \\
\hline $75-79$ & 213 & 74,1 & 235 & 81,7 & 10,3 & 124 & 32,9 & 146 & 38,9 & 18,2 \\
\hline $80 \mathrm{e}+$ & 321 & 89,7 & 354 & 98,8 & 10,1 & 223 & 44,1 & 262 & 51,7 & 17,2 \\
\hline Total & 1266 & 52,1 & 1395 & 57,4 & 10,2 & 722 & 24,1 & 847 & 28,3 & 17,4 \\
\hline
\end{tabular}

O cálculo das reais magnitudes e a precisão das estimativas dos indicadores de mortalidade dessa região têm enfrentado importantes entraves devido à subenumeração dos registros de óbitos e aos problemas na qualidade das informações sobre as causas básicas de óbito. Assim, a utilização de métodos de correção reduzem problemas de subestimação dos níveis de mor- talidade, proporcionando uma melhor qualidade da informação que pode servir mais adequadamente à gestão pública.

Tradicionalmente, tem-se recorrido a técnicas indiretas de mensuração e correção do subregistro de óbitos. Embora tenha sido alvo de muitos debates e discussões, em que pese as divergências entre estimativas do subregistro obtidas pelo 
Tabela 3. Óbitos e taxas de mortalidade padronizada por tipos de câncer antes e após a correção, segundo sexo. Estados do Nordeste, 2010.

\begin{tabular}{|c|c|c|c|c|c|c|c|c|c|c|}
\hline \multirow{4}{*}{ Estado } & \multicolumn{5}{|c|}{ Homens } & \multicolumn{5}{|c|}{ Mulheres } \\
\hline & \multicolumn{2}{|c|}{ Antes } & \multicolumn{2}{|c|}{ Após } & \multirow{2}{*}{$\begin{array}{c}\text { Variação } \\
\text { da taxa }(\%)\end{array}$} & \multicolumn{2}{|c|}{ Antes } & \multicolumn{2}{|c|}{ Após } & \multirow{2}{*}{$\begin{array}{r}\text { Variação } \\
\text { da taxa }(\%\end{array}$} \\
\hline & $\mathbf{n}$ & taxa & $\mathbf{n}$ & taxa & & $\mathbf{n}$ & taxa & $\mathbf{n}$ & taxa & \\
\hline & \multicolumn{5}{|c|}{ Próstata } & \multicolumn{5}{|c|}{ Mama } \\
\hline MA & 270 & 106,1 & 335 & 131,4 & 23,9 & 60 & 20,6 & 119 & 40,6 & 96,5 \\
\hline PI & 203 & 138,4 & 233 & 158,5 & 14,5 & 54 & 30,1 & 81 & 45,2 & 50,2 \\
\hline $\mathrm{CE}$ & 545 & 135,1 & 640 & 158,7 & 17,5 & 230 & 45,3 & 333 & 65,6 & 44,9 \\
\hline RN & 242 & 159,1 & 286 & 187,7 & 18,0 & 74 & 38,2 & 115 & 59,3 & 55,3 \\
\hline $\mathrm{PB}$ & 263 & 131,9 & 313 & 157,4 & 19,3 & 98 & 37,4 & 147 & 56,3 & 50,4 \\
\hline $\mathrm{PE}$ & 615 & 165,6 & 694 & 186,8 & 12,8 & 280 & 52,2 & 374 & 69,7 & 33,7 \\
\hline $\mathrm{AL}$ & 128 & 116,7 & 154 & 140,3 & 20,2 & 52 & 34,8 & 77 & 51,1 & 47,0 \\
\hline SE & 149 & 200,5 & 165 & 222,0 & 10,7 & 56 & 53,9 & 73 & 70,4 & 30,6 \\
\hline BA & 921 & 148,3 & 1170 & 188,3 & 26,9 & 289 & 36,1 & 512 & 63,9 & 77,1 \\
\hline Total & 3337 & 143,2 & 3989 & 171,2 & 19,5 & 1193 & 39,4 & 1831 & 60,5 & 53,4 \\
\hline \multirow[t]{2}{*}{ AT } & 793 & 94,4 & 1016 & 90,6 & 16,2 & 237 & 33,3 & 439 & 29,8 & 65,9 \\
\hline & \multicolumn{10}{|c|}{ Traqueia, brônquios e pulmões } \\
\hline MA & 134 & 49,7 & 186 & 69,2 & 39,3 & 79 & 26,8 & 112 & 38,0 & 41,6 \\
\hline PI & 105 & 69,5 & 129 & 85,4 & 22,9 & 58 & 32,7 & 74 & 41,2 & 26,0 \\
\hline $\mathrm{CE}$ & 335 & 82,9 & 413 & 102,1 & 23,2 & 253 & 50,0 & 311 & 61,5 & 23,1 \\
\hline $\mathrm{RN}$ & 106 & 70,6 & 141 & 94,3 & 33,5 & 76 & 39,2 & 99 & 51,2 & 30,5 \\
\hline $\mathrm{PB}$ & 111 & 56,7 & 152 & 77,8 & 37,2 & 84 & 32,1 & 112 & 42,7 & 33,0 \\
\hline $\mathrm{PE}$ & 303 & 78,1 & 368 & 94,6 & 21,2 & 218 & 40,6 & 271 & 50,5 & 24,4 \\
\hline $\mathrm{AL}$ & 66 & 55,0 & 87 & 72,6 & 31,9 & 41 & 27,0 & 55 & 36,3 & 34,1 \\
\hline SE & 61 & 76,8 & 74 & 93,3 & 21,5 & 38 & 37,0 & 48 & 46,3 & 25,2 \\
\hline BA & 370 & 57,7 & 572 & 89,1 & 54,6 & 223 & 27,9 & 349 & 43,5 & 56,3 \\
\hline Total & 1590 & 66,3 & 2121 & 88,5 & 33,4 & 1070 & 35,4 & 1430 & 47,3 & 33,6 \\
\hline \multirow[t]{2}{*}{ AT } & 309 & 33,2 & 498 & 32,9 & 33,4 & 215 & 23,2 & 301 & 25,2 & 33,2 \\
\hline & \multicolumn{10}{|c|}{ Estômago } \\
\hline MA & 115 & 43,0 & 128 & 47,8 & 11,1 & 47 & 15,9 & 58 & 19,8 & 24,4 \\
\hline PI & 49 & 32,5 & 55 & 36,4 & 11,8 & 32 & 18,1 & 38 & 21,0 & 16,3 \\
\hline $\mathrm{CE}$ & 307 & 76,0 & 326 & 80,7 & 6,2 & 185 & 36,2 & 206 & 40,2 & 10,9 \\
\hline RN & 107 & 71,0 & 115 & 76,7 & 8,1 & 60 & 30,5 & 68 & 34,6 & 13,4 \\
\hline $\mathrm{PB}$ & 111 & 56,7 & 121 & 61,8 & 9,1 & 75 & 28,3 & 85 & 32,0 & 12,8 \\
\hline $\mathrm{PE}$ & 220 & 56,6 & 236 & 60,6 & 7,1 & 124 & 23,0 & 142 & 26,5 & 14,9 \\
\hline $\mathrm{AL}$ & 35 & 29,6 & 40 & 33,9 & 14,6 & 19 & 12,7 & 24 & 15,9 & 25,4 \\
\hline SE & 37 & 47,0 & 41 & 51,0 & 8,5 & 20 & 18,9 & 23 & 22,1 & 17,1 \\
\hline BA & 284 & 44,5 & 334 & 52,2 & 17,3 & 161 & 19,9 & 204 & 25,3 & 27,2 \\
\hline Total & 1266 & 52,9 & 1395 & 58,3 & 10,2 & 722 & 23,7 & 847 & 27,8 & 17,3 \\
\hline AT & 272 & 46,4 & 294 & 46,8 & 11,1 & 166 & 23,5 & 183 & 24,3 & 16,3 \\
\hline
\end{tabular}

IBGE e pela Pesquisa de Busca Ativa ${ }^{20}$, a técnica direta usada por esta última fonte tem se mostrado uma alternativa viável para reduzir o efeito do subregistro nas estimativas de indicadores de mortalidade ${ }^{10}$. Por exemplo, em 2010, o número de óbitos de idosos resgatados pela Pesquisa de Busca Ativa totalizaram 20.249 óbitos para o Nordeste, que correspondeu a um aumento de $12 \%$ em relação aos óbitos notificados pelo SIM no mesmo ano. Vale destacar que esse número foi superior aos óbitos notificados como causas mal definidas, que totalizaram 15.592 óbitos.

$\mathrm{O}$ estudo de Borges $^{20}$ mostrou que as estimativas de esperança de vida, ao nascer no ano 2010, para as Unidades da Federação, utilizando os óbitos do SIM corrigidos pela Pesquisa de Busca Ativa, apresentaram valores mais altos que os publicados pelo IBGE, indicando diferenças 
nas estimativas de cobertura dos óbitos. Ademais, as esperanças de vida calculadas pelo IBGE se mostraram correlacionadas com variáveis socioeconômicas, sendo mais altas nas Unidades da Federação com melhores condições socioeconômicas, como apontado pela extensa literatura sobre o tema. Por outro lado, as esperanças de vida calculadas com base nos dados da Pesquisa de Busca Ativa apresentaram fraca correlação, inclusive na direção oposta do esperado para alguns indicadores. Desse modo, chama-se atenção para a necessidade de mais trabalhos de avaliação da Pesquisa de Busca Ativa, com discussão de seus alcances e limitações ${ }^{21}$.

A contribuição dada pela Pesquisa de Busca Ativa foi superior aos códigos garbage neste estudo. No entanto, o somatório dos óbitos recuperados proporcionou um aumento importante nas magnitudes das mortes dos idosos do Nordeste por câncer. Considerando ambos os sexos, houve uma redistribuição de 6.229 óbitos no total. Destes, $2.435(39,1 \%)$ foram atribuídos ao conjunto dos principais cânceres masculinos e femininos enfatizados neste estudo. Os estados com maior recuperação dos dados para ambos os sexos foram, em ordem decrescente: Bahia, Ceará e Pernambuco, já que são os mais populosos ${ }^{22}$.

Embora os códigos garbage tivessem um impacto reduzido, pois se restringiram a redistribuição de apenas dois códigos (C76 e C80) por serem específicos do câncer, estudos mais gerais observaram um impacto maior desses códigos e, portanto, apontaram a necessidade de considerar não apenas o Capítulo XVIII (códigos R), mas também os códigos garbage de outros capítulos da CID-10 na avaliação da qualidade das informações sobre causas de óbito ${ }^{12,23,24}$.

Neste estudo, o câncer da traqueia, brônquios e pulmões apresentou níveis de distribuição diferentes segundo sexo, com taxas mais elevadas nos homens idosos que nas mulheres idosas. Malta et al. ${ }^{10}$ constataram maiores taxas de mortalidade por este tipo de câncer nos homens de 30 a 69 anos que nas mulheres dessa faixa etária no Brasil e regiões, em 2011. A maioria dos estudos sobre o tema justifica este fato pela distinta exposição ao tabagismo entre homens e mulheres ${ }^{25}$.

Para a Região Nordeste, os resultados do presente estudo quando confrontados com os achados de Malta et al..$^{10}$ revelam um efeito maior da redistribuição dos óbitos nas taxas de mortalidade por câncer de traqueia, brônquios e pulmões em idosos em relação ao grupo de 30 a 69 anos, pois, enquanto a variação das taxas antes e após a correção de subregistro, redistribuição de causas mal definidas e códigos garbage para o grupo de 30 a 69 anos foi de $21 \%$ para homens e $20 \%$ para mulheres, a variação para os idosos ficou em cerca de $33 \%$ em cada sexo.

É interessante notar que antes da correção dos óbitos, o câncer de traqueia, brônquios e pulmões apresentou as maiores taxas de mortalidade feminina nos estados do Maranhão, Piauí, Ceará e Rio Grande do Norte e o câncer de mama nos demais estados. Entretanto, após a correção, o câncer de mama liderou a mortalidade da população idosa feminina em todos os estados nordestinos. Ou seja, a mudança de status hierárquico permitiu homogeneizar um comportamento em todo o Nordeste. Este reposicionamento serve como sinalização para o estabelecimento de prioridades nas políticas de saúde pública da região. Se o câncer de mama não era a primeira causa para os estados mencionados e, após a correção, passou a ser, então é possível atribuir a mudança de status aos erros de inconsistência dos registros básicos de óbitos originais. Ressalta-se que Maranhão tem a pior cobertura de óbitos do País ${ }^{26}$.

A idade é um fator preponderante na mortalidade por câncer, uma vez que as maiores taxas correspondem às idades mais avançadas. Este fato é preocupante, pois em detrimento do fenômeno do envelhecimento populacional os níveis da morbimortalidade de idosos pelos principais tipos de câncer tendem a aumentar. Para se ter ideia da dimensão, o aumento da mortalidade proporcional por câncer desse grupo etário passou de 53,5\% em 1979 para $68,4 \%$ em $2015^{4}$.

É aviltante o aumento das taxas de mortalidade por câncer de próstata dos 60 aos 80 anos e mais no Nordeste em 2010, com cerca de 18 vezes nestes extremos. Com uma taxa de 557,4 óbitos por 100.000 idosos após a correção, significa que para cada 100.000 idosos com 80 anos ou mais, 557 morreram de câncer de próstata. Antes da correção a taxa era de 467, também acentuada. Consequentemente, os erros nos registros dos óbitos podem se tornar mais sérios à medida que aumenta a quantidade de idosos em idades mais avançadas. Ishitani et al. ${ }^{24}$ reforçam este comportamento ao observarem que as maiores proporções de códigos gargabe foram verificadas em crianças de 1 a 4 anos e em idosos acima dos 60 anos para o período de 2011-2013 em Belo Horizonte-MG.

Comparando o panorama da mortalidade por câncer de idosos do Nordeste em 2010 com os dados mais recentes divulgados pelo DATASUS, referentes ao ano de 2015, constatou-se que as neoplasias da próstata, da traqueia, brônquios e pulmões e do estômago continuam, nessa ordem, 
sendo as três principais causas da mortalidade de homens por câncer. Em 2015, essas neoplasias foram responsáveis por $23,5 \%, 12,8 \%$ e $8,5 \%$ dos óbitos de idosos por neoplasias, respectivamente. Porém, houve aumento na proporção de óbitos por câncer de traqueia, brônquios e pulmões e redução nas proporções de próstata e estômago. Já em relação ao sexo feminino, em 2015, os três primeiros lugares foram ocupados pelas neoplasias da traqueia, brônquios e pulmões $(11,8 \%)$, da mama $(11,7 \%)$ e do colorretal $(7,2 \%)$. Vale destacar o aumento no percentual de óbitos por câncer da traqueia, brônquios e pulmões, que era de 10,6\%, em 2010, e, em 2015, atingiu os níveis do câncer de mama. Este, por sua vez, com um percentual de $11,1 \%$, em 2010, apresentou um percentual bem próximo ao de 2015.

Em nível mundial, apesar de expressiva sua magnitude, o número de pessoas que morrem por determinado tipo de câncer varia muito ao redor do globo. Na análise da Agência Internacional de Pesquisas sobre o Câncer (IARC), os cânceres de pulmão, próstata, estômago e colorretal estiveram entre os principais protagonistas da mortalidade por câncer de homens nos diversos continentes, em 2012. Em relação à mortalidade das mulheres, o estudo apontou altas taxas de câncer de mama, pulmão, colorretal, colo do útero e estômago dentre os principais ${ }^{27}$.

\section{Colaboradores}

JB Carvalho e NA Paes participaram da concepção do estudo, revisão de literatura, delineamento da metodologia, geração dos resultados, interpretação dos dados e redação. NA Paes realizou a revisão final do artigo.
Em se tratando de um grupo específico de causas de óbito (câncer de próstata, mama, pulmão e estômago) considerado aqui, é de se esperar que a replicação deste estudo para outras causas de óbito da CID-10 seja ainda mais impactante devido ao maior volume de óbitos a redistribuir. Desse modo, é possível que algumas configurações do perfil das causas e magnitudes dos óbitos no Nordeste estejam expostas a vieses e precisariam ser examinados com cautela antes de serem feitos diagnósticos sobre o comportamento do perfil de mortes dos idosos.

Os resultados deste estudo mostraram que a correção dos dados de óbitos significou melhorias na qualidade dos dados, com consideráveis acréscimos na quantidade de óbitos registrados para os principais tipos de câncer. Este fato chama a atenção dos gestores para os verdadeiros níveis de mortalidade da doença ao lidarem com ações de planejamento na saúde pública e epidemiológica. Assim, por serem mais altos do que aqueles registrados, aumenta-se o alerta para que sejam tomadas medidas que perpassem pelo reforço no treinamento dos médicos para o preenchimento correto da declaração de óbito, visando maior acurácia das estatísticas de mortalidade por câncer. Desse modo, auxilia-se nas ações específicas para o idoso e a população em geral. 


\section{Referências}

1. Bray F, Jemal A, Grey N, Ferlay J, Forman D. Global cancer transitions according to the Human Development Index (2008-2030): a population-based study. Lancet 2012; 13(8):790-801.

2. Ferlay J, Soerjomataram I, Ervik M, Dikshit R, Eser S, Mathers C, Rebelo M, Parkin DM, Forman D, Bray F. GLOBOCAN 2012: Estimated Cancer Incidence, Mortality and Prevalence Worldwide in 2012 v1.0 [serial on the Internet]. IARC Cancer Base No. 112013 [acessado 2017 Maio 22]. Available from: http://www. globocan.iarc.fr

3. Torre LA, Bray F, Siegel RL, Ferlay J, Lortet-Tieulent J, Jemal A. Global cancer statistics, 2012. CA Cancer J Clin 2015; 65(2):87-108.

4. Brasil. Ministério da Saúde (MS). SIM - Sistema de Informação sobre Mortalidade. Brasília (DF), 2017. [acessado 2017 Jun 14]. Disponível em: http://www2.datasus.gov.br/DATASUS/index.php?area $=0205 \& i d=6937$

5. Carvalho JAM, Brito F. A demografia brasileira e o declínio da fecundidade no Brasil: contribuições, equívocos e silêncios. Rev bras Est Pop 2013; 22(2):351-369.

6. World Health Organization (WHO). Relatório Mundial sobre Envelhecimento e Saúde [Internet]. Geneva: WHO; 2015. [acessado 2016 Nov 7]. Disponível em: http://www.who.int/ageing/publications/world-report-2015/en/

7. Barbosa IR, Souza DL, Bernal MM, Costa I. Cancer mortality in Brazil: temporal trends and predictions for the year 2030. Medicine (Baltimore) 2015; 94(16):1-6.

8. Santos JP, Paes NA. Associação entre condições de vida e vulnerabilidade com a mortalidade por doenças cardiovasculares de homens idosos do Nordeste do Brasil. Rev Bras Epidemiol 2014; 17(2):407-420.

9. França E, Teixeira R, Ishitani L, Duncan BB, Cortez -Escalante JJ, Morais Neto OL, Szwarcwald CL. Causas mal definidas de óbito no Brasil: método de redistribuição baseado na investigação do óbito. Rev Saude Publica 2014; 48(4):671-681.

10. Malta DC, Abreu DM, Moura LD, Lana GC, Azevedo G, França E. Tendências das taxas de mortalidade de câncer de pulmão corrigidas no Brasil e regiões. Rev Saude Publica 2016; 50:33.

11. Murray CJ, Lopez AD. Mortality by cause for eight regions of the world: Global Burden of Disease Study. Lancet 1997; 349(9061):1269-1276.

12. Naghavi M, Makela S, Foreman K, O’Brien J, Pourmalek F, Lozano R. Algorithms for enhancing public health utility of national causes-of-death data. Popul Health Metr 2010; 8:9.

13. Jorge MHPM, Laurenti R, Lima-Costa MF, Gotlieb SLD, Chiavegatto Filho ADP. A mortalidade de idosos no Brasil: a questão das causas mal definidas. Epidemiol Serv Saúde 2008; 17(4):271-281.

14. Paes NA, Gouveia JF. Recuperação das principais causas de morte no Nordeste do Brasil: impacto na expectativa de vida. Rev Saude Publica 2010; 44(2):301-309.
15. Szwarcwald CL, Morais-Neto OL, Frias PG, SouzaJúnior PRB, Escalante JJC, Lima RB, Viola RC. Busca ativa de óbitos e nascimentos no nordeste e na Amazônia Legal: estimação das coberturas do SIM e do SINASC nos municípios brasileiros. In: Brasil. Ministério da Saúde (MS). Secretaria de Vigilância em Saúde. Departamento de Análise de Situação de Saúde. Saúde Brasil 2010: uma análise da situação de saúde e de evidências selecionadas de impacto de ações de vigilância em saúde. Brasília: MS; 2011. p. 79-98.

16. Cascão AM, Costa AJL, Kale PL. Qualidade da informação sobre mortalidade numa coorte de diabéticos - Estado do Rio de Janeiro, 2000 a 2003. Rev Bras Epidemiol 2012; 15(1):134-142.

17. Hill K, Queiroz B. Ajustando o método da equação geral de balanceamento para migração. Rev Bras Est Pop 2013; 27(1):7-20.

18. Ledermann S. La repartition des decés de causes indeterminées. Revue d'Institut International de Statistique 1955; 23(3):47-57.

19. Kanso S, Romero DE, Leite IC, Moraes EN. Diferenciais geográficos, socioeconômicos e demográfi$\cos$ da qualidade da informação da causa básica de morte dos idosos no Brasil. Cad Saude Publica 2011; 27(7):1323-1339.

20. Borges GM. Avaliação das estimativas oficiais de cobertura de óbitos e dos indicadores de mortalidade no Brasil. VII Congresso de la Asociación Latinoamericana de Población e XX Encontro Nacional de Estudos Populacionais, Foz do Iguaçu/PR; Brasil; 2016.

21. Borges GM, Silva LO. Fontes de dados de fecundidade no Brasil: características, vantagens e limitações. In: Ervatti LR, Borges GM, Jardim AP, organizadores. Mudança demográfica no Brasil no início do século XXI: subsídios para as projeções da população. Rio de Janeiro: IBGE; 2015. p. 10-29.

22. Instituto Brasileiro de Geografia e Estatística (IBGE). Censo Demográfico 2010 [Internet]. [acessado 2018 Jan 24]. Disponível em: https://cidades.ibge.gov.br/ brasil/ba/pesquisa/ 23/25207?tipo=ranking

23. GBD 2015 Mortality and Causes of Death Collaborators. Global, regional, national life expectancy, allcause mortality, and cause specific mortality for 249 causes of death, 1980-2015: a systematic analysis for the Global Burden of Disease Study 2015. Lancet 2016; 388(10053):459-544.

24. Ishitani LH, Teixeira RA, Abreu DMX, Paixão LMMM, França EB. Qualidade da informação das estatísticas de mortalidade: códigos garbage declarados como causas de morte em Belo Horizonte, 2011-2013. Rev bras Epidemiol 2017; 20(1):34-45.

25. Levi F, Lucchini F, Negri E, Zatonski W, Boyle P, La Vecchia C. Trends in cancer mortality in the European Union and accession countries. Ann Oncol 2004; 15(9):1425-1431. 
26. Brasil. Ministério da Saúde (MS). RIPSA - Rede Interagencial de Informações para a Saúde. Indicadores e Dados Básicos [Internet]. Brasília: MS; 2018. [acessado 2018 Fev 08]. Disponível em: http://tabnet.datasus. gov.br/cgi/idb2012/a1801b.htm

27. May M. Statistics: Attacking an epidemic. Nature [serial on the Internet] 2014 [acessado 2017 Mar 22]; 509(7502):S50-S51. Available from: http://dx.doi. org/10.1038/509S50a

Artigo apresentado em 01/09/2017

Aprovado em 24/03/2018

Versão final apresentada em 26/03/2018 